\title{
Synergistic interactions of the anti-casein kinase 2 CIGB-300 peptide and chemotherapeutic agents in lung and cervical preclinical cancer models
}

\author{
YASSER PERERA $^{1}$, NEYLEN DEL TORO ${ }^{1}$, LARISA GOROVAYA ${ }^{2}$, \\ JORGE FERNANDEZ-DE-COSSIO ${ }^{3}$, HERNAN G. FARINA ${ }^{4}$ and SILVIO E. PEREA ${ }^{1}$
}

\begin{abstract}
${ }^{1}$ Laboratory of Molecular Oncology, Division of Pharmaceuticals; ${ }^{2}$ Animal Facility Unit; ${ }^{3}$ Department of Bioinformatics, Center for Genetic Engineering and Biotechnology (CIGB), Havana 10600, Cuba; ${ }^{4}$ Laboratory of Molecular Oncology, Quilmes National University, Bernal, Buenos Aires B1876BXD, Argentina
\end{abstract}

Received March 27, 2014; Accepted May 13, 2014

DOI: $10.3892 / \mathrm{mco} .2014 .338$

\begin{abstract}
CIGB-300 is a novel clinical-stage synthetic peptide that impairs the casein kinase 2 (CK2)-mediated phosphorylation of B23/nucleophosmin in different experimental settings and cancer models. As a single agent, CIGB-300 induces apoptosis in vitro and in vivo and modulates an array of proteins that are mainly involved in drug resistance, cell proliferation and apoptosis, as determined by proteomic analysis. However, the clinical oncology practice and cumulative knowledge on tumor biology suggest that drug combinations are more likely to cope with tumor complexity compared to single agents. In this study, we investigated the antiproliferative effect of CIGB-300 when combined with different anticancer drugs, such as cisplatin (alkylating), paclitaxel (antimitotic), doxorubicin (antitopoisomerase II) or 5-fluorouracil (DNA/RNA antimetabolite) in cell lines derived from lung and cervical cancer. Of note, using a Latin square design and subsequent analysis by CalcuSyn software, we observed that paclitaxel and cisplatin exhibited the best synergistic/additive profile when combined with CIGB-300, according to the combination and dose reduction indices. Such therapeutically favorable profiles may be explained by a direct cytotoxic effect and also by the observed cell cycle impairment following incubation of tumor cells with selected drug combinations. Importantly, on in vivo dose-finding schedules in human cervical tumors xenografted in nude mice, we observed that concomitant administration of CIGB-300 and cisplatin increased mice survival compared to single-agent treatment. Collectively, these findings provide a rationale for combining the
\end{abstract}

Correspondence to: Dr Yasser Perera, Laboratory of Molecular Oncology, Division of Pharmaceuticals, Center for Genetic Engineering and Biotechnology (CIGB), P.O. Box 6162, Havana 10600, Cuba

E-mail: yasser.perera@cigb.edu.cu

Key words: casein kinase 2, CIGB-300, drug combination, synergism, chemotherapy
anti-CK2 CIGB-300 peptide with currently available anticancer agents in the clinical setting and indicate platins and taxanes as compounds with major perspectives.

\section{Introduction}

The implication of constitutive and often exacerbated enzymatic activity of casein kinase 2 (CK2) in the maintenance of the malignant phenotype has been firmly established by in vitro and in vivo preclinical experimentation and epidemiological findings in several types of tumors $(1,2)$. Based on such knowledge, different groups are currently engaged in the quest for highly potent and specific CK2 inhibitors, which may overcome the limitations of first-generation anti-CK2 compounds, such as 5,6-dichloro-1-( $\beta$-D-ribofuranosyl)-1Hbenzimidazole (DRB), 4,5,6,7-tetrabromo-1H-benzimidazole, 4,5,6,7-tetrabromo-1H-benzotriazole (TBB) and 2-dimethylamino-4,5,6,7-tetrabromo-1H-benzimidazole (3-8). Such new-generation anti-CK2 compounds are ultimately represented by CX-4945, a small-molecule adenosine triphosphate (ATP) competitor currently in clinical cancer trials $(9,10)$. Focusing on the same tumor-specific CK2 signaling but aiming to abrogate such events by binding to the phosphoaceptor domain on its substrates, the CIGB-300 peptide was discovered by screening a phage display library against a model CK2 substrate (11).

In different experimental settings and preclinical cancer models, CIGB-300 was shown to inhibit the CK2-mediated phosphorylation of the validated CK2 substrate B23/nucleophosmin, leading to a fast cell death by apoptosis $(12,13)$. However, considering the high degree of conservation for the CK2 phosphoaceptor domain, a multitarget effect may be anticipated (14). Such a multitarget effect may better explain the diverse arrays of proteins and processes that appear to be modulated by CIGB-300 and its already established antiangiogenic effect $(15,16)$. Of note, CIGB-300 exerts a broad antiproliferative effect on cell lines derived from breast, cervical, lung, colon and prostate cancer, while a robust antitumor effect was also observed in vivo in mouse models of cervical and lung cancer $(13,17,18)$. In the clinical setting, CIGB-300 was 
investigated in a phase I clinical trial on high-grade squamous intraepithelial lesions (HSILs), establishing its safety and tolerability by local injection (19). More recently, another phase I clinical study on patients with stage 1B2/II cervical cancer allowed us to estimate the maximum tolerated dose (MTD) and the pharmacokinetics/biodistribution profiles for CIGB-300 following local administration (20). Moreover, a phase I study on lung and haematological cancers further demonstrated that CIGB-300 may also be administered by intravenous injection without significant toxicity (unpublished data). Of note, the first evidence regarding the antitumor effect of CIGB-300 in humans was recently collected from case studies (21).

Despite these promising preclinical and clinical findings using the CIGB-300 peptide, the clinical oncology setting and cumulative knowledge on cancer biology suggest that drug combinations are more likely to cope with tumor complexity compared to single agents (22). Of note, previous data from proteomics studies demonstrated that CIGB-300 modulates a group of proteins directly involved in anticancer drug resistance (15). Therefore, in this study, we evaluated the antiproliferative effect of CIGB-300 when combined with four different chemotherapeutics drugs in two model cell lines derived from lung and cervical cancer. With the aim to select the optimal combination in these two clinically relevant tumor types, the peptide was combined in a pairwise manner with anticancer drugs, such as antimitotic (paclitaxel), alkylating (cisplatin), antitopoisomerase II (doxorubicin) or DNA/RNA antimetabolite (5-fluorouracil) agents. Based on the estimation of the combination index (CI) (23), all the interactions were classified as synergistic, additive or antagonistic and the direct effect on cell viability and proliferation was analyzed. Finally, the potential benefit of one selected drug combination over each monotherapy was corroborated in vivo in a mouse model of cervical cancer.

\section{Materials and methods}

Cell lines and chemotherapeutic agents. The NCI-H125 non-small-lung cancer cell line and the SiHa cervix-derived squamous carcinoma cell line were originally acquired from American Type Culture Collection (Manassas, VA, USA) and cultured in RPMI or Dulbecco's modified Eagle's medium supplemented with $10 \%$ fetal bovine serum, unless otherwise stated. The clinical grade chemotherapeutic drugs paclitaxel, cisplatin, doxorubicin and 5-fluorouracil (Drug Research and Development Center, Havana, Cuba) were kindly provided by the Oncology Service of the National Institute of Oncology and Radiobiology (Havana, Cuba) or from the Hermanos Ameijeiras Hospital (Havana, Cuba).

CIGB-300. The CIGB-300 peptide was synthesized on solid phase, purified by reverse-phase high-performance liquid chromatography to $>98 \%$ purity on acetonitrile $/ \mathrm{H}_{2} \mathrm{O}$-trifluoroacetic acid gradient and confirmed by ion-spray mass spectrometry (Micromass, Manchester, UK).

Drug combination assays. The 48-h sulforhodamine B (SRB)-based assay was adopted from the National Cancer Institute to measure the cytotoxic effect of each drug combination (24). Sulforhodamine B sodium salt was purchased from Sigma-Aldrich (St. Louis, MO, USA). NCI-H125 or SiHa cell suspensions $\left(6 \times 10^{4}\right.$ cells $\left./ \mathrm{ml}\right)$ were seeded on 96 -well plates (Corning Costar; Sigma-Aldrich, ) and on the next day, a curve of serial dilutions for each compound (1:2 or 1:10 on RPMI) were added and incubated for $48 \mathrm{~h}$. Following plate reading, the data were analyzed by the CalcuSyn software, which is based on the median effect equation $(23,25)$. The type of interaction was scored as synergistic, additive or antagonistic, according to the obtained CI. The interaction maps were represented by color-coded surfaces rendering a $3 \mathrm{D}$ visual effect within a $2 \mathrm{D}$ plot. The maps were built by superimposing filled contours (CI) and isoline plots (Fa, fraction affected), partially scripted with Matlab ${ }^{\circledR}$ R2012a software (7.14.0.0739; http://www.mathworks.com/).

Cytotoxicity by propidium iodide (PI) exclusion. NCI-H125 or $\mathrm{SiHa}$ cells were seeded at $6 \times 10^{4}$ cells $/ \mathrm{ml}$ on 24 -well culture plates (Corning Costar) and incubated for $24 \mathrm{~h}$. On the next day, selected concentrations of CIGB-300, paclitaxel, cisplatin, or their combinations, were added to the plates and incubated for $0.5,2,5,8,24$ and $36 \mathrm{~h}$. The cells were subsequently washed with cold phosphate-buffered saline (PBS) and stained for $10 \mathrm{~min}$ with $5 \mu \mathrm{g} / \mathrm{ml}$ PI solution. Finally, the stained cells were analyzed on the FL2 channel using the Partec PAS III particle analyzing system flow cytometer and their proprietary FloMax v2.4f software (Partec GmbH, Münster, Germany).

Cell cycle analysis. The cell cultures were incubated with selected concentrations of CIGB-300, paclitaxel, cisplatin, or their combinations for $24 \mathrm{~h}$. Subsequently, the cells were collected by trypsinization, washed and fixed with ice-cold methanol/acetone $(4: 1)$ at $4^{\circ} \mathrm{C}$ for $1 \mathrm{~h}$. The cells were then stained by incubation for $20 \mathrm{~min}$ at $37^{\circ} \mathrm{C}$ with a solution of $100 \mathrm{mg} / \mathrm{ml}$ PI and $10 \mathrm{mg} / \mathrm{ml}$ of DNase-free RNase. Finally, the stained cells were analyzed on the abovementioned PAS III flow cytometer using the cell cycle analyzer from the FloMax software. Prior to fitting the DNA distribution to a diploid DNA content for cell cycle profiling, cellular debris and doublets were properly excluded by gating out in FL3-A vs. FL3-W two-parameter dot plots.

\section{In vivo experiments.}

Scheme A. A total of 28, 8-week-old male nude mice (Harlan Laboratories $\mathrm{GmbH}$, Eystrup, Germany/National Atomic Energy Commission, Buenos Aires, Argentina) were injected subcutaneously in the right flank with $4 \times 10^{6} \mathrm{SiHa}$ cells in $300 \mu \mathrm{l}$ PBS. When the tumors reached $30 \mathrm{~mm}^{3}$, the mice were randomly assigned into 7 groups ( $n=4$ per group) and were administered placebo, CIGB-300 (days 1-5, 50 or $200 \mu \mathrm{g}$ ), cisplatin (days 1, 3 and 5, 1 or $4 \mathrm{mg} / \mathrm{kg}$ ), or their combinations, by intraperitoneal (cisplatin) or intratumoral (CIGB-300) routes.

Scheme B. A total of 35 8-week-old female nude mice (Harlan Laboratories GmbH/National Atomic Energy Commission) were injected with $3 \times 10^{6} \mathrm{SiHa}$ cells as described for scheme A. When the tumors reached $30 \mathrm{~mm}^{3}$, the mice were randomly assigned into 7 groups ( $n=5$ per group) and injected with placebo, CIGB-300 (days 1-5, 100 or $200 \mu \mathrm{g}$ ), cisplatin (days 1,3 and 5,3 or $6 \mathrm{mg} / \mathrm{kg}$ ), or their combinations, 
Table I. Drug potency $\left(\mathrm{IC}_{50}\right)$ and steepness $(\mathrm{m})$ of the dose-response curves obtained after fitting the SRB-based assay data to the median effect equation using the CalcuSyn software.

\begin{tabular}{|c|c|c|c|c|c|c|c|}
\hline \multirow[b]{2}{*}{ Compound } & \multirow[b]{2}{*}{ Class } & \multicolumn{3}{|c|}{ NCI-H125 cells } & \multicolumn{3}{|c|}{ SiHa cells } \\
\hline & & $\begin{array}{r}\mathrm{IC}_{50} \\
(\mu \mathrm{M})\end{array}$ & $\mathrm{m}$ & $\begin{array}{c}\text { Drug range } \\
\qquad(\mu \mathrm{M})\end{array}$ & $\begin{array}{r}\mathrm{IC}_{50} \\
(\mu \mathrm{M})\end{array}$ & $\mathrm{m}$ & $\begin{array}{l}\text { Drug range } \\
\quad(\mu \mathrm{M})\end{array}$ \\
\hline CIGB-300 & CK2-targeted therapy & 69.80 & 1.60 & $6.25-200$ & 91.10 & 1.17 & $12.5-400$ \\
\hline Paclitaxel & Antimitotic & 0.03 & 0.13 & $0.00002-2$ & 0.01 & 0.13 & $0.0002-2$ \\
\hline Cisplatin & Alkylating & 9.43 & 2.38 & $0.31-10$ & 6.61 & 0.96 & $1.56-25$ \\
\hline Doxorubicin & Antitopoisomerase II & 0.14 & 0.76 & $0.00025-25$ & 0.13 & 0.35 & $0.00025-25$ \\
\hline 5-Fluorouracil & RNA/DNA antimetabolite & 17.83 & 0.40 & $0.02-2000$ & 1.28 & 0.11 & $0.02-200$ \\
\hline
\end{tabular}

The classification of each anticancer compound and the selected range of concentrations for further combinations experiments are also shown. $\mathrm{SRB}$, sulforhodamine $\mathrm{B}$; CK2, casein kinase 2 ; $\mathrm{IC}_{50}$, half maximal inhibitory concentration.

A
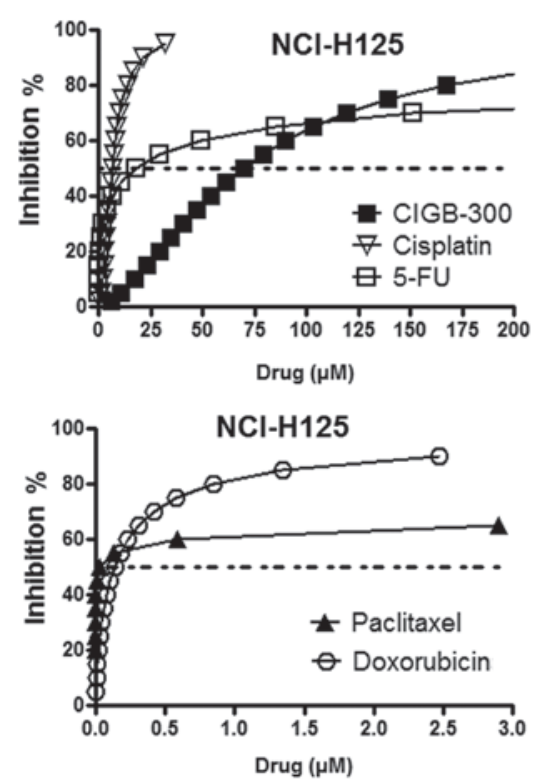

B
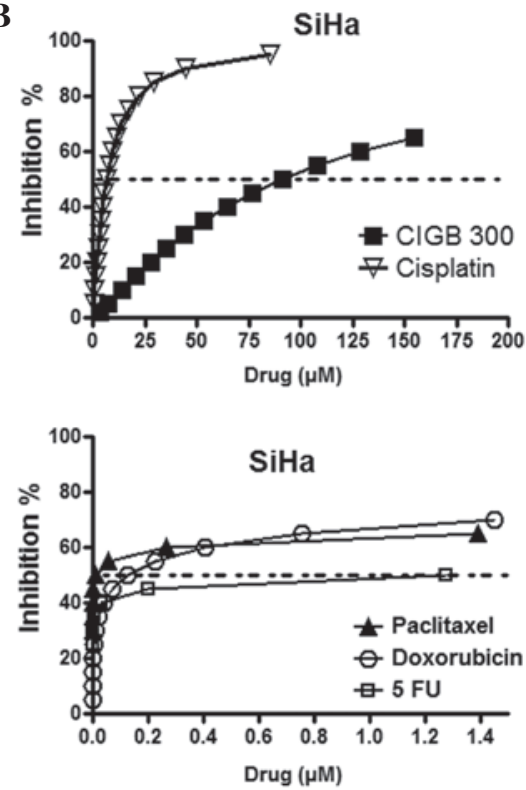

Figure 1. Antiproliferative dose-response curves for CIGB-300 and chemotherapeutic drugs assessed as single agents in the (A) NCI-H125 lung cancer and (B) SiHa cervical cancer cell lines. The curves were generated by fitting the data derived from the sulforhodamine B-based assays to the median effect equation using the CalcuSyn software. The quality of the fitting was assessed by the r coefficient, where an r $>0.9$ was obtained for all curves. 5-FU, 5-fluorouracil.

by the routes indicated in scheme A. The tumors were measured every other day with a caliper and their volumes were calculated as follows: volume $=$ length $\mathrm{x}$ width ${ }^{2} / 2$. Survival was daily registered during the experimentation, unless the tumor volumes reached $2,000 \mathrm{~mm}^{3}$, in which case the animals were sacrificed due to ethical considerations. The mice were maintained under pathogen-free conditions and all the procedures were performed in accordance with the recommendations for the proper use and care of laboratory animals at the Center for Genetic Engineering and Biotechnology (Havana, Cuba).

Statistical analysis. The tumor volumes among the different groups were compared using one-way analysis of variance (ANOVA) and Tukey's post hoc test. The log-rank test was applied for the survival analysis of the Kaplan-Meier curves. All the statistical analyses were performed using GraphPad
Prism version 4.00 for Windows (GraphPad Software, San Diego CA, USA).

\section{Results}

Antiproliferative effect of CIGB-300 and chemotherapeutic drugs as single agents. In order to select a suitable range of drug concentrations for combination experiments, we first evaluated the antiproliferative effect of CIGB-300 and four different chemotherapeutics drugs used in the treatment of lung (paclitaxel and cisplatin) and cervical cancer (paclitaxel, cisplatin, doxorubicin and 5-fluorouracil) with the SRB-based assay (Fig. 1).

The fitting of each curve to the median effect equation allowed us to estimate the half maximal inhibitory drug concentration $\left(\mathrm{IC}_{50}\right)$, also referred to as drug potency, and the steepness or shape of the curve for each individual 
A

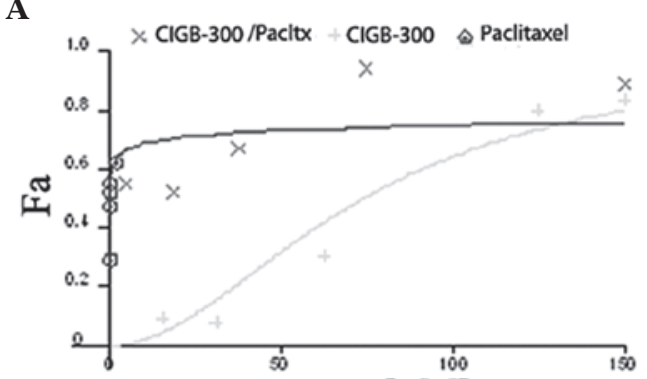

C

Drug $[\mu \mathrm{M}]$

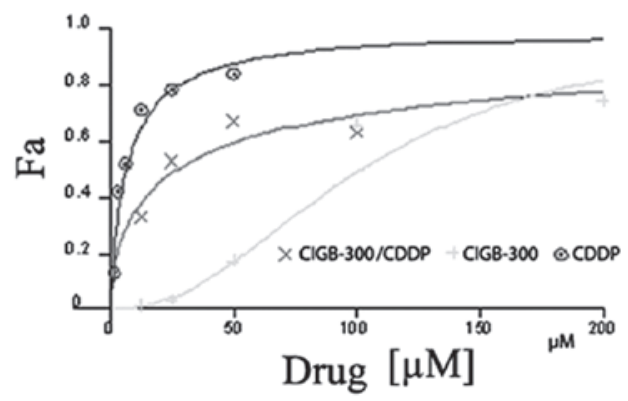

B
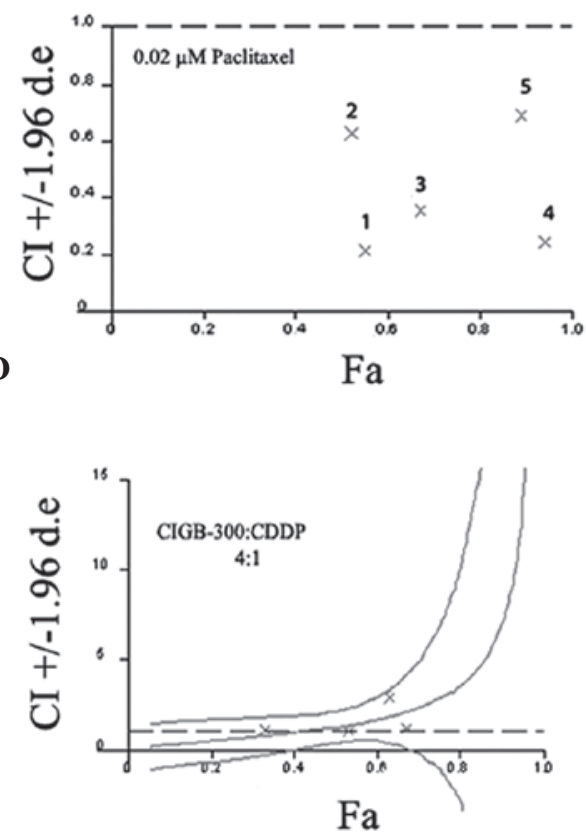

Figure 2. Graphs generated by the CalcuSyn software using the fraction affected (Fa) vs. concentration data for each drug alone or its combinations in the NCI-H125 (CIGB-300 plus paclitaxel) and SiHa (CIGB-300 plus cisplatin) cell lines. (A and C) Dose-response curves obtained for each drug or its combinations at non-constant ratios using a fixed concentration of paclitaxel $(0.02 \mu \mathrm{M})$ in NCI-H125 cells (A), or at constant ratios among the drugs (CIGB-300:cisplatin, 4:1) in SiHa cells (C). (B and D) CI vs. Fa graphs for the combinations described in (A and C), respectively. Pacltx, paclitaxel; CDDP, cisplatin; CI, combination index.

drug (m) (Table I). As expected, due to the diverse nature and mechanism of action of the tested compounds, the results demonstrated that these drugs differ in both parameters. While CIGB-300 exerts a major effect at $>50 \mu \mathrm{M}$ in both cell lines, the majority of the chemotherapeutic drugs exert a significant effect on cell proliferation at $<5 \mu \mathrm{M}$. The flat sigmoidal shape $(m<1)$ of certain drugs, such as paclitaxel, doxorubicin and 5-fluorouracil, prompted us to use serial 10-fold dilutions in the combination experiments to cover an extensive range of cytotoxic effects.

Drug combination experiments. To determine the type of interaction (i.e., synergistic, additive or antagonistic) between the CIGB-300 peptide and conventional chemotherapeutic drugs in the two tumor types, we used the CalcuSyn software. A Latin square design was selected to investigate such interactions across a range of different drug concentrations, which included clinically achievable drug levels. Representative examples of the graphs generated from the analysis of two particular drug combinations, i.e., CIGB-300 plus paclitaxel in NCI-H125 cells (non-constant ratio combinations) and CIGB-300 plus cisplatin in SiHa cells (constant-ratio combinations), are shown in Fig. 2.

To visualize the results obtained from all 8 combination experiments (i.e., CIGB-300 pairwise combined with 4 drugs in 2 cell lines), an interaction surface was built by representing in a color code the estimated CI for each particular pair of drug combinations (i.e., 6x6 concentration matrix) (Figs. 3 and 4). Moreover, in order to assess the actual relevance of the observed interaction pattern, we also represented the $\mathrm{Fa}$ by the combinations over this $2 \mathrm{D}$ graphical display of interaction data.

The results of the combination experiments in the SiHa cervical cancer cell line demonstrated that CIGB-300 interacts synergistically with paclitaxel in $>50 \%$ of the interaction surface, with effect levels or $\mathrm{Fa}>60 \%$ of the cell population, while only small areas of antagonism were registered at extreme concentrations (i.e., low for both drugs or very high for paclitaxel) (Fig. 3A). A similar but less promising interaction pattern was observed for the combination of CIGB-300 plus doxorubicin, where regions of additive or slight antagonism between the two drugs were found to be more predominant at higher effect levels $(>50 \%)$ when the concentration of doxorubicin increased (Fig. 3C). An intermediary scenario was observed for the combination of CIGB-300 plus cisplatin, with $>70 \%$ of the interaction surface exhibiting an additive pattern even at high effect levels (40-85\%) (Fig. 3B). The worse combination scenario was clearly observed for the CIGB-300 plus 5-fluorouracil combination, where a strong antagonism was observed in approximately half of the interaction surface, with synergistic interaction areas only seen at very high concentrations of both drugs (Fig. 3D).

In the NCI-H125 lung cancer cell line, the combination of CIGB-300 plus paclitaxel exhibited the best synergistic profile, with $>75 \%$ of the interaction surface displaying a $\mathrm{CI}<0.7$ and effect levels $>50 \%$ (Fig. 4A). Of note, a similar interaction pattern in lung and cervical cancer cells was observed for the combination of CIGB-300 plus cisplatin, with significant areas of the interaction surface exhibiting an additive pattern at effect levels $>60 \%$ (Fig. 4B). Finally, a moderate (CI $>1.20)$ to strong antagonism $(\mathrm{CI}>3.3)$ was observed practically across the entire interaction surface when the combinations of CIGB-300 plus doxorubicin and CIGB-300 plus 5-fluorouracil were evaluated in NCI-H125 cells (Fig. 4C and D).

Another important output derived from the analysis of drug interaction data using CalcuSyn is the dose reduction 
A
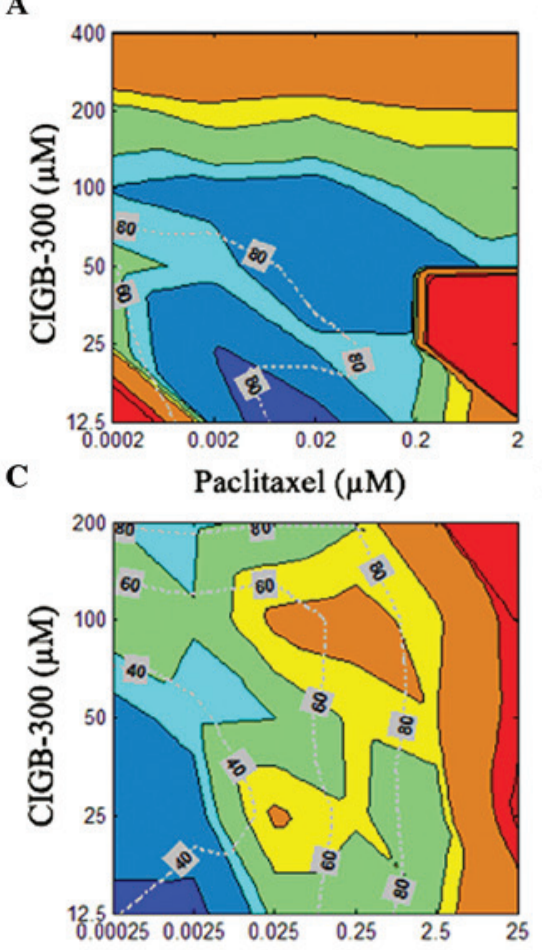
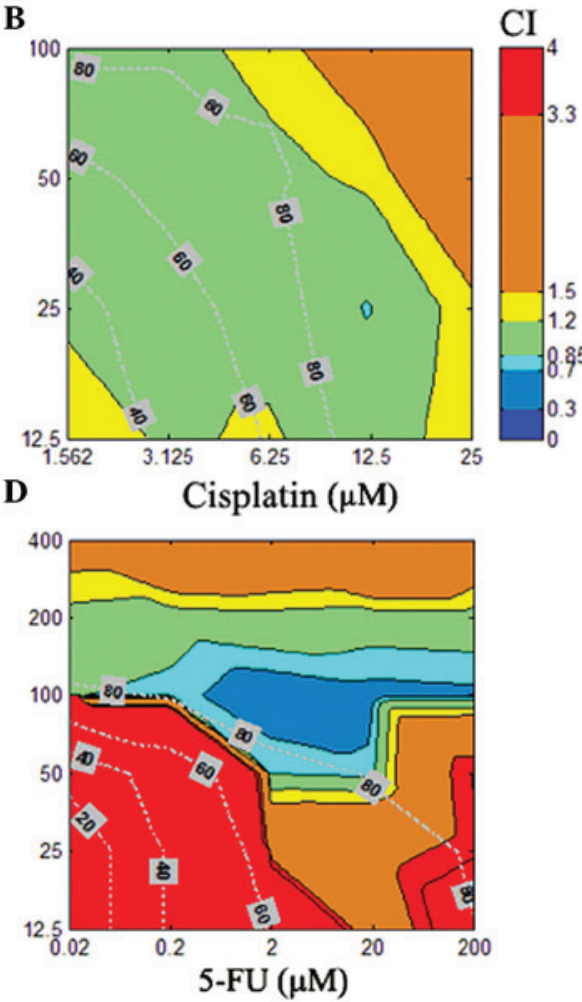

Figure 3. Interaction surface built using the estimated combination index (CI) for each combination experiment comprising the CIGB-300 peptide and the chemotherapeutic drugs (A) paclitaxel, (B) cisplatin, (C) doxorubicin, or (D) 5-fluorouracil (5-FU) in the SiHa cervical cancer cell line. The CI was calculated with the CalcuSyn software for each particular drug combination and represented in 2D color code graphics or interaction surface. The color code spans from strong synergism (dark blue, $\mathrm{CI}<0.30$ ) to strong antagonism (red, CI $>3.3$ ). The graphics also include the fraction affected (Fa; expressed in \%) by each combination across such an interaction surface (dotted line on top). The concentrations of each particular drug in the combinations are denoted on each axis.

A

C
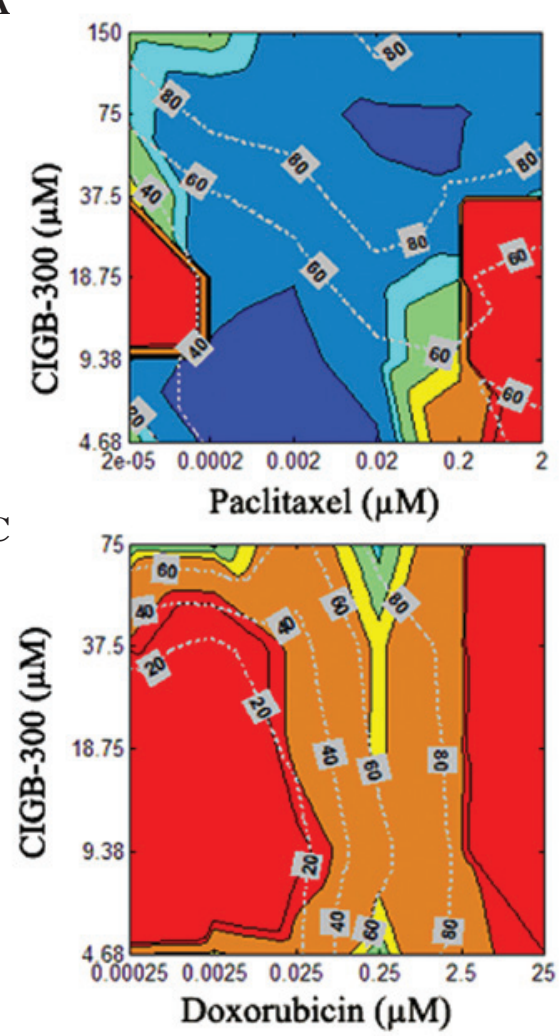
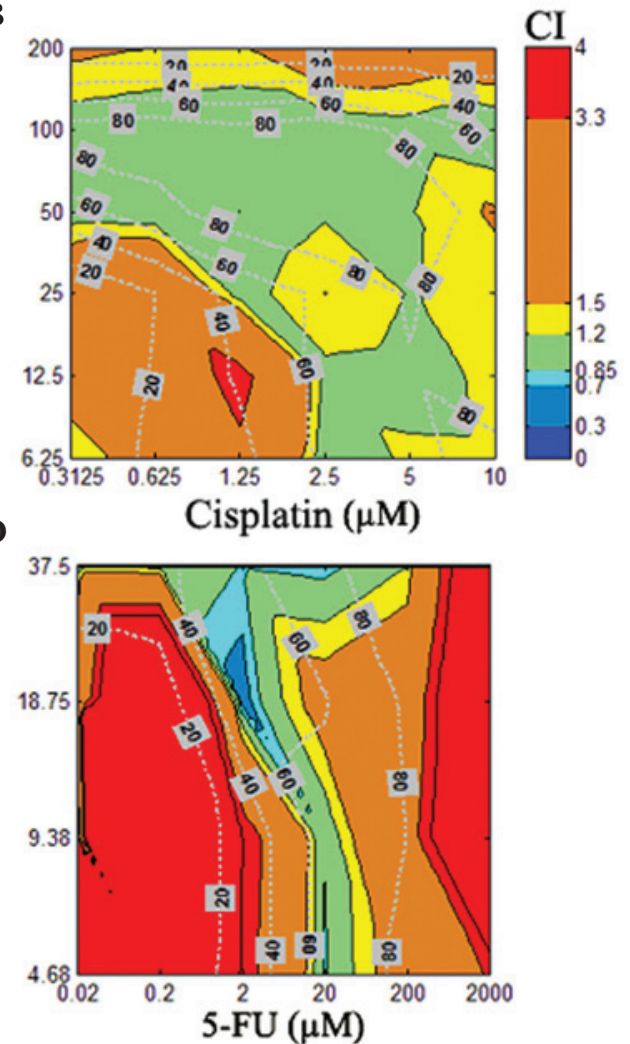

Figure 4. Interaction surface built using the estimated combination index (CI) for each combination experiment comprising the CIGB-300 peptide and the chemotherapeutic drugs (A) paclitaxel, (B) cisplatin, (C) doxorubicin, or (D) 5-fluorouracil (5-FU) in the NCI-H125 lung cancer cell line. The graphics show the fraction affected $(\mathrm{Fa})$ by each combination across such an interaction surface and the concentrations of each particular drug in the combinations. 
Table II. Parameters estimated using the CalcuSyn software for two selected combinations of CIGB-300 plus chemotherapy in NCI-H125 and SiHa cells.

\begin{tabular}{|c|c|c|c|c|c|c|c|c|}
\hline \multirow[b]{2}{*}{ Cell line } & \multicolumn{2}{|l|}{ Combination } & \multicolumn{3}{|c|}{ Parameters } & \multirow[b]{2}{*}{$\begin{array}{l}\text { Interaction } \\
\text { type }\end{array}$} & \multicolumn{2}{|c|}{ DRI } \\
\hline & Drugs & $(\mu \mathrm{M})$ & $\begin{array}{l}\mathrm{IC}_{50} \\
(\mu \mathrm{M})\end{array}$ & CI & $\mathrm{Fa}$ & & Drug 1 & Drug 2 \\
\hline NCI-H125 & CIGB-300 + paclitaxel & $75+0.02$ & 0.03 & 0.30 & 0.94 & Strong synergism & 5.3 & 3250 \\
\hline $\mathrm{SiHa}$ & CIGB-300 + CDDP & $25+12.5$ & 6.61 & 0.83 & 0.73 & Moderate synergism & 8.5 & 1.2 \\
\hline
\end{tabular}

CDDP, cisplatin; $\mathrm{IC}_{50}$, half maximal inhibitory concentration; CI, combination index; Fa, fraction affected; DRI, dose reduction index .

A
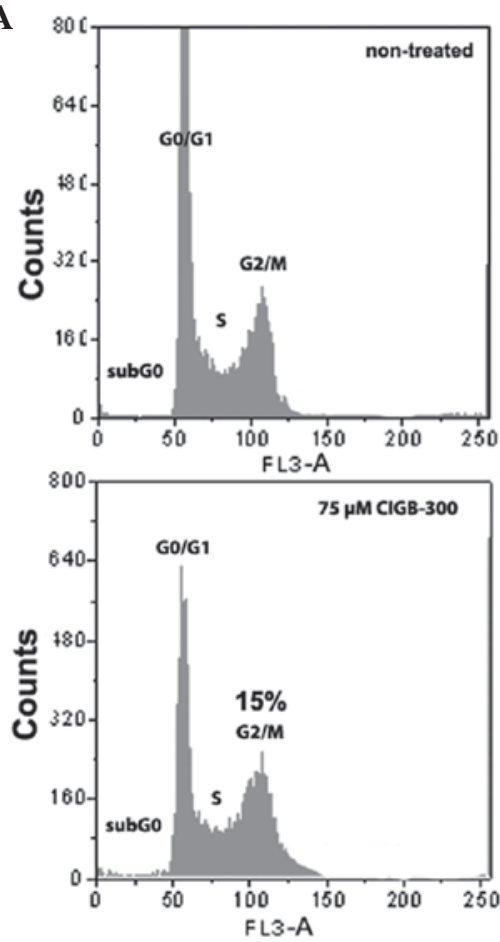

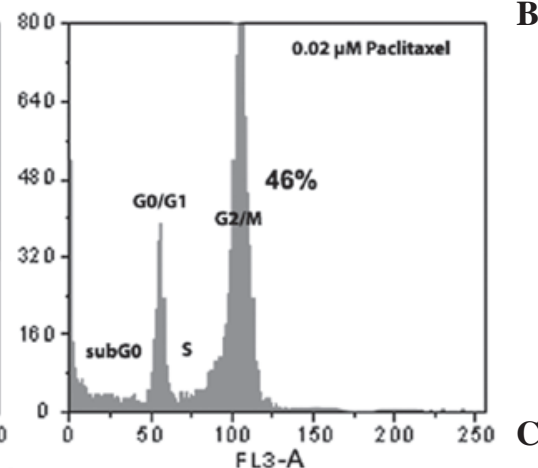

B
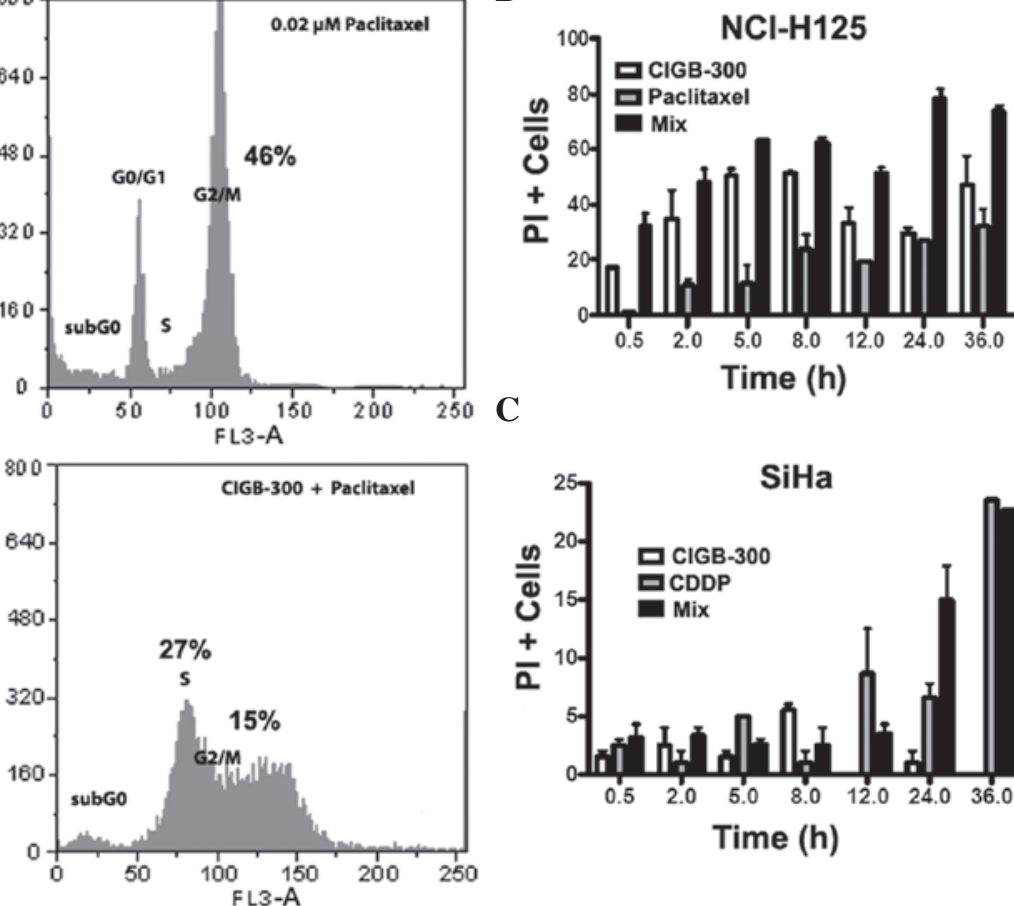

$\mathrm{SiHa}$

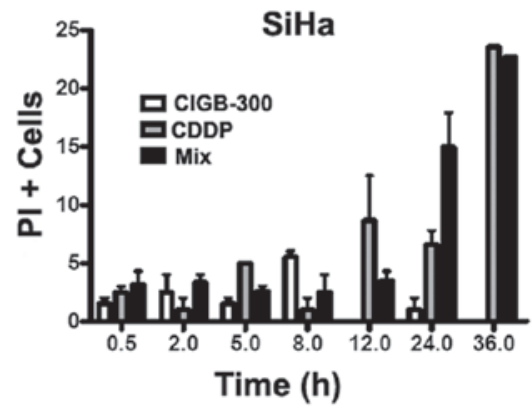

Figure 5. Effect of selected drug combinations on (A) cell cycle progression and (B and C) cell viability of NCI-H125 and SiHa cells. (A) The DNA content from treated and untreated NCI-H125 cells was assessed by flow cytometry using the FloMax software. The percentages refer to the difference between non-treated and treated cells at particular cell cycle phases (normalization). (B and C) Cytotoxicity was determined by flow cytometry using propidiun iodide (PI) as a vital stain. NCI-H125 or SiHa cells were incubated for 0.5-36 h with each drug alone or its combinations at the concentrations described in Table II; the cells were then stained with PI and analyzed by flow cytometry.

index (DRI). This parameter is inversely associated with CI and represents the number of times each single drug may be reduced in a combination setting without compromising the final therapeutic effect (23). The estimated DRI from the combination of CIGB-300 plus paclitaxel indicated that, in a synergistic scenario like those exemplified in Table II, 5-fold less peptide (DRI=5.3) is required to achieve an antiproliferative effect level of $94 \%$ in NCI-H125 cells $(\mathrm{Fa}=0.94)$. Furthermore, in the same combination setting, the concentration of paclitaxel may be reduced 3,250 times without compromising the final antiproliferative effect in lung cancer cells (Table II). A significant DRI was also achieved in the combination of CIGB-300 plus cisplatin for the peptide (DRI=8.5) but not for cisplatin, where only a small DRI was observed (DRI=1.2) (Table II). As expected, in the majority of the combination experiments where a synergistic pattern was observed, important DRIs were registered for all the drugs (data not shown).

Effect of drug combinations on cell cycle and viability. The characterization of the type of interaction performed thus far was based on a global proliferative readout (i.e., cell mass staining) from the SRB-based assay. To evaluate how selected drug combinations affect cell proliferation and viability, we measured the DNA content and the loss of cytoplasmic membrane integrity as indicators of cell cycle progression and cell cytotoxicity, respectively. Two particular synergistic drug combinations, namely CIGB-300 plus paclitaxel in lung cancer cells and CIGB-300 plus cisplatin in cervical cancer cells, were selected for the analysis (Table II, Figs. 3 and 4).

The effect of the combination of CIGB-300 plus paclitaxel on cell cycle progression in lung cancer cells was determined 
A
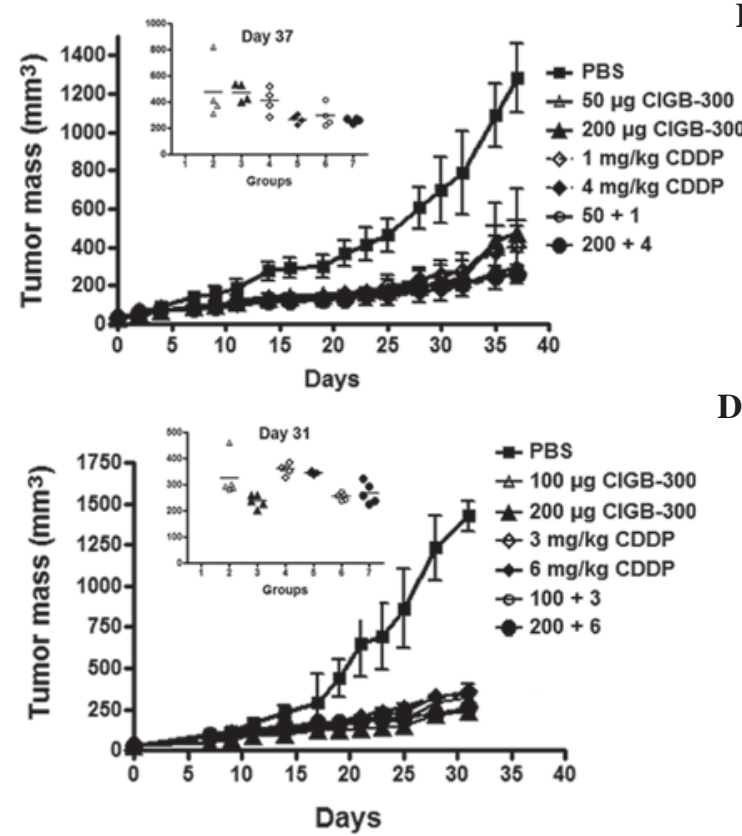

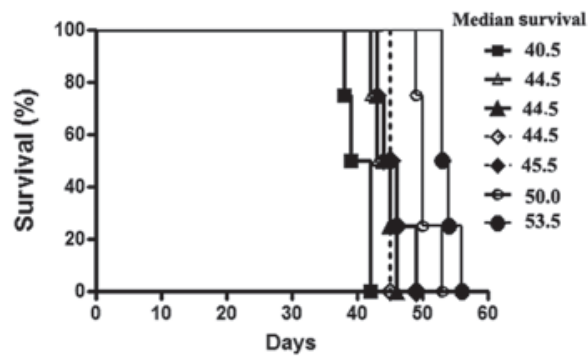

D

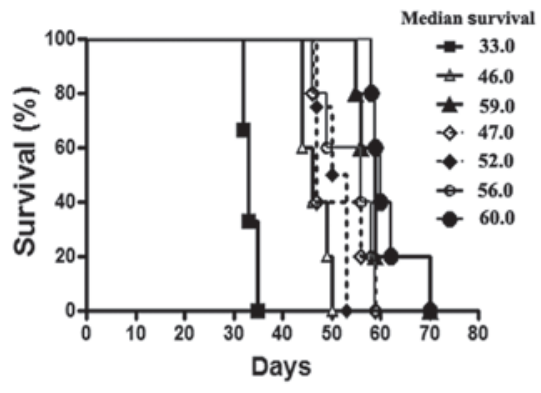

Figure 6. Antitumor effect of CIGB-300, cisplatin (CDDP) or their combinations, in a cervical cancer model based on the inoculation of SiHa cells in nude mice. Once the tumor mass reached $30 \mathrm{~mm}^{3}$ (day 0), the mice were randomized into groups and injected with CIGB-300, cisplatin or their combinations at indicated doses, according to schemes A and B described in Materials and methods. (A and B) Tumor growth and Kaplan-Meier survival curves obtained from treated animals in scheme A. (C and D) Tumor growth and survival curves obtained from treated animals in scheme B.

using PI to stain genomic DNA. After gating out cellular debris and excluding cell doublets, the DNA distribution was fitted to a diploid DNA content for cell cycle profiling. The analysis demonstrated that both CIGB-300 and paclitaxel cause a cell cycle arrest in the $\mathrm{G} 2 / \mathrm{M}$ phase of the cell cycle (15 and 46\%, respectively), while their combination caused cell cycle arrest at the $\mathrm{S}(27 \%)$ and $\mathrm{G} 2 / \mathrm{M}(15 \%)$ phases (Fig. 5A). Of note, in SiHa cells, CIGB-300 at $25 \mu \mathrm{M}$ did not affect the cell cycle distribution, whereas cisplatin and the combination caused only a mild arrest at the S phase (10\%) (data not shown).

As regards the cytotoxicity of the selected drug combinations, the results obtained with the combination of CIGB-300 plus paclitaxel in lung cancer cells corroborated the previously observed synergistic interaction. We observed a stronger cytotoxic effect for the combination compared to the one registered for each drug alone, which was more evident after 24 and $36 \mathrm{~h}$ of incubation (Fig. 5B). This kinetic experiment also revealed that, while paclitaxel-induced cytotoxicity increases gradually from 2 to $36 \mathrm{~h}$, the effect of CIGB-300 may be seen as early as $0.5 \mathrm{~h}$, reaching a maximum effect between 5 and $8 \mathrm{~h}$ and after 36 h. Finally, the cytotoxic effect of CIGB-300 alone at $25 \mu \mathrm{M}$ was found to be negligible in the SiHa cervical cancer cell line compared to that of cisplatin alone or its combination with CIGB-300 (Fig. 5C).

In vivo drug combination in a cervical cancer animal model. To corroborate the potential benefit of combining the CIGB-300 with chemotherapy in vivo, we evaluated the effect of simultaneous administration of the peptide and cisplatin on tumor growth and survival following the inoculation of $\mathrm{SiHa}$ cells in nude mice. In both experiments (schemes $\mathrm{A}$ and $\mathrm{B}$ ), the doses for each single agent and its combinations were selected to maintain a fixed ratio among the drugs to further evaluate the type of interaction by CalcuSyn. In scheme A (CIGB-300:cisplatin, 2.5:1), the single agents as well as their combination significantly reduced tumor growth (>60\% inhibition, T/C $0.4-0.2$, day 37) and increased the survival of treated mice (4-13 days) in this cervical cancer model (Fig. 6A and B)

Of note, in this experimental setting, the lower dose combination of CIGB-300 plus cisplatin achieved a more significant tumor growth delay and survival compared to each monotherapy alone (Fig. 6A insert and Fig. 6B). At day 37, the group treated with this combination (CIGB-300 $50 \mu \mathrm{g}$ plus cisplatin $1 \mathrm{mg} / \mathrm{kg}$ ) resulted in $77 \%$ tumor growth inhibition $(\mathrm{T} / \mathrm{C}=0.23)$, while the corresponding monotherapies achieved a less significant effect (CIGB-300: $63 \%$ inhibition, $\mathrm{T} / \mathrm{C}=0.37$; and cisplatin: $68 \%$ inhibition, $\mathrm{T} / \mathrm{C}=0.32$ ) (Fig. $6 \mathrm{~A}$ insert). However, no significant differences were observed between such groups (one-way ANOVA, Tukey's post hoc test). The superiority regarding the tumor growth inhibition of combining the higher doses of each compound (CIGB-300 $200 \mu \mathrm{g}$ plus cisplatin $4 \mathrm{mg} / \mathrm{kg}$ ) was rather subtle, as a consequence of the strong effect of cisplatin alone (80\% vs. $79 \%$, respectively) (Fig. 6A). By contrast, the survival analysis indicated that the combination of the lower doses of CIGB-300 and cisplatin significantly increased the survival (5.5 days) of tumor-bearing mice compared to each monotherapy alone (combination vs. CIGB-300, $\mathrm{P}<0.05$; combination vs. cisplatin, $\mathrm{P}<0.01$ ), whereas the higher dose combination prolonged survival up to 8 days over each single agent (combination vs. CIGB-300, $\mathrm{P}<0.01$; combination vs. cisplatin, $\mathrm{P}<0.01)$ (log-rank test) (Fig. 6B).

In scheme B (CIGB-300:cisplatin, 1.7:1), although both single agents and their combinations exerted a potent antitumor 
effect (>70\% inhibition, T/C 0.25-0.17, day 31), the differences in tumor growth inhibition for monotherapies compared to combination treatments did not reach statistical significance (Fig. 6C insert). However, the survival analysis indicated that the lower dose combination (CIGB-300 $100 \mu \mathrm{g}$ plus cisplatin $3 \mathrm{mg} / \mathrm{kg}$ ) prolonged mouse survival at least by 9 days compared to each monotherapy alone $(\mathrm{P}<0.05)$ (Fig. 6D).

\section{Discussion}

CIGB-300 is a novel clinical stage molecule designed to impair CK2-mediated phosphorylation by binding to the conserved phosphoaceptor domain on its substrates (11). The antineoplastic effect of this peptide has been well documented in the preclinical setting in vitro and in vivo $(13,17,18)$. In 2006 , the peptide entered a phase I clinical trial on patients with HSILs, where safety and efficacy signs were registered (19). Moreover, in patients with stage 1B2/II cervical cancer, the MTD, biodistribution and modulation of a response biomarker were investigated following local administration of CIGB-300 (20). Finally, evidence of the antitumor activity of CIGB-300 in humans was recently collected from case studies (21).

Although preclinical and clinical findings support the use of the CIGB-300 peptide as a monotherapy, it is widely accepted that the combination of drugs in the field of oncology are more likely to cope with tumor complexity compared to single-agent treatment (22). Such a view is sustained by decades of clinical practice and by cumulative knowledge on tumor biology (26). Drug combination therapies are commonly used in order to increase therapeutic efficacy, reduce toxicity and decrease the incidence of drug resistance. In this scenario, the combination of targeted therapies plus conventional chemotherapy has emerged as an alternative therapeutic option that requires further investigation (27).

To the best of our knowledge, the antineoplastic effect of the CIGB-300 peptide when combined with different chemotherapeutic drugs currently used in the treatment of lung and cervical cancer was first investigated in this study. The drugs used included antimitotic and DNA alkylating agents, topoisomerase II inhibitors and DNA/RNA antimetabolites, hence targeting different global cellular processes. In order to avoid false claims of synergism commonly found in the literature, we performed our interaction studies using the CalcuSyn software, which is based on the median effect equation described by Chou (23). One of the main advantages of this analysis over the classical isobologram is that the type of interaction can be scored through an entire range of drug combinations and effect levels once a Latin square design is selected. Of note, in order to visualize all interaction data at the same time, a simple script was built to draw an interaction surface. On such an interaction surface, additional representation of the effect level allowed us to better assess the relevance of the observed interaction pattern 'at a first glance'. Indeed, synergistic interactions at low effect levels (e.g., $\mathrm{Fa}<0.4)$ may be less relevant compared to slight synergism or even additive interaction at higher effect levels (e.g., Fa>80), since the complete eradication of cancer cells is the final therapeutic goal in the clinical oncology setting.

Our in vitro interaction studies suggested that the anti-mitotic drug paclitaxel may be a suitable partner to combine with CIGB-300 in lung and cervical malignancies. A plain synergistic interaction was observed in most of the interaction surface from NCI-H125 and SiHa cells. Importantly, such synergistic interaction resulted in $>70 \%$ proliferation inhibition $(\mathrm{Fa}>0.70)$ in both cell lines. Moreover, since CI and DRI are inversely associated, such a 'positive' interaction suggests that, each drug in the combination may be significantly reduced without compromising the final therapeutic outcome, hence limiting the chances of unwanted side effects. This issue is of paramount importance in the case of paclitaxel, a drug known to be associated with significant toxicities, such as myelosuppression and peripheral neuropathy (28).

The combination of CIGB-300 with the alkylating drug cisplatin displayed an additive interaction pattern across most of the interaction surface in SiHa cells. Of note, for paclitaxel and cisplatin, further combination experiments demonstrated that the simultaneous addition of CIGB-300 and the chemotherapeutic agent is more favorable compared to their sequential addition, according to schedule-dependent experiments (data not shown). Altogether, while the combination of the CIGB-300 peptide with the antimitotic drug paclitaxel appears to be more favorable for lung cancer treatment, the combination of CIGB-300 with cisplatin may be more promissory in cervical cancer. Considering that paclitaxel and cisplatin are chemotherapeutic drugs currently used for the treatment of lung and cervical cancer, respectively, the evaluation of these particular drug combinations in their already approved clinical niches may be more expedite. However, a quite different profile was obtained from combining CIGB-300 with doxorubicin in lung and cervical cancer cells. In lung cancer cells, the combination of CIGB-300 with the topoisomerase II inhibitor and with DNA/RNA antimetabolites, resulted in antagonistic surfaces with rather scarce areas of additive or synergistic effects under these experimental conditions.

A further layer of analysis in drug combination experiments is whether drug concentrations resulting in a synergistic interaction are actually achievable in vivo. The pharmacokinetic analysis of CIGB-300 in animal models and humans indicated that this peptide may reach concentrations up to $30 \mu \mathrm{M}$ in the plasma, whereas after a 3-h infusion of paclitaxel at doses of $135-225 \mathrm{mg} / \mathrm{m}^{2}$ the estimated Cmax ranged between 3.3 and 7.6 $\mu \mathrm{M}$. (unpublished results; 29). According to such reported values, a significant area of the synergistic interaction between these two drugs occurs in the range of clinically achievable concentrations. Importantly, a similar analysis also suggested that the predominant additive profile obtained from the combination of CIGB-300 plus cisplatin in cervical cancer cells occurs in a concentration range achievable in vivo for CIGB-300 $(<30 \mu \mathrm{M})$ and cisplatin $(<15 \mu \mathrm{M})(30,31)$.

Considering that in the in vivo setting precise drug concentrations or ratios are difficult to achieve (32), broad favorable interaction surfaces in vitro (i.e., synergistic or additive) may suggest a higher chance of obtaining an antitumor effect at the organism level, both in mouse models and hopefully the clinical setting. Likewise, such favorable profiles provide us with more confidence to exclude potential antagonism arising from drug combinations at particular ratios or concentrations. Following this rationale and taking into account that cervical cancer is one of the clinical niches where CIGB-300 is currently being evaluated as a monotherapy (20), we attempted to corroborate 
the potential therapeutic benefit of combining CIGB-300 with cisplatin in vivo after inoculating human cervical cancer cells in nude mice. Although such experiments were initially designed to be analyzed by CalcuSyn, the reduction of tumor growth with monotherapies and drug combinations were far above the $\mathrm{IC}_{50}$ in all cases $(\mathrm{T} / \mathrm{C}<0.5)$, thus precluding the generation of reliable dose-response curves for CI determination (23). However, our results demonstrated that two different CIGB-300 plus cisplatin combinations (i.e., different doses and drug ratios) significantly increased animal survival when compared to each monotherapy alone. Such in vivo results are in complete accordance with the broad additive interaction surface observed with the concentration range and drug ratios evaluated in vitro in the antiproliferative setting.

Although drug combination experiments may be performed over days or weeks, the elucidation of the cellular and molecular basis underlying the observed interactions typically requires years of research. The analysis at the cellular level for one particular drug combination (i.e., CIGB-300 plus paclitaxel) in lung cancer cells indicated that the observed synergistic interaction arose from their effect on cell cycle progression and viability. However, only cytotoxicity appeared to contribute to the observed synergism regarding the combination of CIGB-300 plus cisplatin in cervical cancer cells, suggesting that particular drug combinations affect cell proliferation at different levels.

The first molecular clues that may explain the synergistic interaction with anticancer drugs in lung cancer cells were previously revealed by proteomics analysis of CIGB-300-treated NCI-H125 cells (15). In such studies, the peptide modulated an array of proteins associated with drug resistance and survival, which may favor the cytotoxic effect of several chemotherapeutic drugs. One of the downregulated proteins was found to be the nuclease-sensitive element-binding protein 1 (YB-1), a transcriptional factor that activates the expression of the multidrug resistance 1 protein (MDR1) in response to genotoxic stress (33). MDR1 pumps out a myriad of cytotoxic compounds, including paclitaxel; therefore, a putative decrease in MDR1 expression via YB-1 may explain the increased sensitivity of tumor cells to paclitaxel (34). Furthermore, YB-1 participates in the nucleotide excision repair pathway and plays a role in cisplatin-DNA adduct reparation (35); therefore, its downregulation may also account for the observed additive pattern in the combination of CIGB-300 plus cisplatin. Intriguingly, although YB-1 is a validated substrate for CK2 (36), this protein does not interact with CIGB-300 according to previous pull-down experiments conducted on lung cancer cells (12).

CIGB-300 was also shown to decrease the levels of glutathione S-transferase Pi (GST-Pi), a cytoplasmic detoxifying enzyme, whose increased levels correlate with resistance to a wide range of drugs (37). Although paclitaxel and cisplatin are not direct substrates for this enzyme, both drugs require the activation of the MAP kinase pathway to exert their maximal cytotoxicity, which is prevented by high levels of GST-Pi. Moreover, at least three other proteins associated with drug resistance were clearly downregulated by CIGB-300 in NCI-H125 cells (15). Whether the synergistic/additive interaction patterns observed with the combination of CIGB-300 with cisplatin or paclitaxel may be explained by these molecular events require elucidation by further studies.
Despite the availability of a significant number of studies on CK2, the number of reports on the use of CK2 inhibitors in combination with anticancer compounds remains limited (38). Such pioneering studies mainly rely on the genetic manipulation of the catalytic subunit CK2 $\alpha$ or the use of the first -generation semi-selective CK2 inhibitors DRB and TBB (38). Although these former studies provided valuable preclinical data, only with the advent of clinical-grade inhibitors the true potential of combining anti-CK2 approaches with conventional or emerging therapies may be translated into clinical grounds. Apart from the CIGB-300 peptide, only the ATP-competitive inhibitor CX-4945 has been evaluated in humans as an anti-CK2 therapy $(9,10)$. A synergistic pattern for particular drug concentrations or ratios was reported when CX-4945 was combined with different compounds targeting the phosphatidylinositol 3 kinase (PI3K)/Akt/mammalian target of rapamycin (mTOR) signaling axis in breast or lung preclinical cancer models $(39,40)$. Considering that CK2 phosphorylates critical substrates in this pathway (e.g., Akt, phosphatase and tensin homolog, p70SK6), the rationale behind such combination experiments was to reinforce the inhibitory effect of compounds such as erlotinib (epithelial growth factor receptor), LY294002 (PI3K) or the dual inhibitor PI-103 (PI3K and mTOR). Moreover, the combination of CX-4945 with cisplatin also resulted in a synergistic drug interaction in two ovarian cancer cell lines according to the Bliss additivity method $(41,42)$. Beyond the fact that the methods of drug interaction analysis differ, those studies only evaluated particular drug concentrations in vitro, without providing an explanation for its selection or investigating a wide range of concentrations for each of the compounds $(39,41)$.

As a novel approach to impair the CK2-mediated signaling, we investigated the potential therapeutic benefit of combining the CIGB-300 peptide with different cytotoxic compounds targeting global cellular processes, which are firmly established in clinical oncology. Although we are currently focused on the elucidation of the molecular and cellular basis of observed synergistic/additive interactions, the preclinical data provided in this study may pave the way for near-future clinical trials investigating the combination of the CIGB-300 peptide with conventional chemotherapeutic agents, such as paclitaxel and cisplatin in two frequent human cancers.

\section{Acknowledgements}

This study was supported by C.I.G.B. and Biorec. Grant CIGB-300.

\section{References}

1. Guerra B and Issinger OG: Protein kinase CK2 in human diseases. Curr Med Chem 15: 1870-1886, 2008

2. Ruzzene M and Pinna LA: Addiction to protein kinase CK2: a common denominator of diverse cancer cells? Biochim Biophys Acta 1804: 499-504, 2010.

3. Zandomeni R, Zandomeni MC, Shugar D and Weinmann R: Casein kinase type II is involved in the inhibition by 5,6-dichloro-1-beta-D-ribofuranosylbenzimidazole of specific RNA polymerase II transcription. J Biol Chem 261: 3414-3419, 1986.

4. Szyszka R, Grankowski N, Felczak K and Shugar D: Halogenated benzimidazoles and benzotriazoles as selective inhibitors of protein kinases CK I and CK II from Saccharomyces cerevisiae and other sources. Biochem Biophys Res Commun 208: 418-424, 1995. 
5. Sarno S, Reddy H, Meggio F, et al: Selectivity of 4,5,6,7-tetrabromobenzotriazole, an ATP site-directed inhibitor of protein kinase CK2 ('casein kinase-2'). FEBS Lett 496: 44-48, 2001.

6. Pagano MA, Meggio F, Ruzzene M, Andrzejewska M, Kazimierczuk $\mathrm{Z}$ and Pinna LA: 2-Dimethylamino-4,5,6,7-tetrabromo-1H-benzimidazole: a novel powerful and selective inhibitor of protein kinase CK2. Biochem Biophys Res Commun 321: 1040-1044, 2004.

7. Duncan JS, Gyenis L, Lenehan J, Bretner M, Graves LM, Haystead TA and Litchfield DW: An unbiased evaluation of CK2 inhibitors by chemoproteomics: characterization of inhibitor effects on CK2 and identification of novel inhibitor targets. Mol Cell Proteomics 7: 1077-1088, 2008.

8. Pagano MA, Bain J, Kazimierczuk Z, et al: The selectivity of inhibitors of protein kinase CK2: an update. Biochem J 415: 353-365, 2008

9. Marschke RF, Andreopoulou E, Von Hoff DD, Lim JK, Padgett CS and Northfelt DW: Phase I clinical trial of CX-4945: A first-in-class orally administered small molecule inhibitor of protein kinase CK2. Mol Cancer Ther 8: C39, 2009.

10. Siddiqui-Jain A, Drygin D, Streiner N, et al: CX-4945, an orally bioavailable selective inhibitor of protein kinase CK2, inhibits prosurvival and angiogenic signaling and exhibits antitumor efficacy. Cancer Res 70: 10288-10298, 2010.

11. Perea SE, Reyes O, Puchades Y, et al: Antitumor effect of a novel proapoptotic peptide that impairs the phosphorylation by the protein kinase 2 (casein kinase 2). Cancer Res 64: 7127-7129, 2004

12. Perera Y, Farina HG, Gil J, et al: Anticancer peptide CIGB-300 binds to nucleophosmin/B23, impairs its CK2-mediated phosphorylation, and leads to apoptosis through its nucleolar disassembly activity. Mol Cancer Ther 8: 1189-1196, 2009.

13. Perera Y, Costales HC, Diaz Y, et al: Sensitivity of tumor cells towards CIGB-300 anticancer peptide relies on its nucleolar localization. J Pept Sci 18: 215-223, 2012.

14. Meggio F and Pinna LA: One-thousand-and-one substrates of protein kinase CK2? FASEB J 17: 349-368, 2003.

15. Rodriguez-Ulloa A, Ramos Y, Gil J, et al: Proteomic profile regulated by the anticancer peptide CIGB-300 in non-small cell lung cancer (NSCLC) cells. J Proteome Res 9: 5473-5483, 2010.

16. Farina HG, Benavent Acero F, Perera Y, et al: CIGB-300, a proapoptotic peptide, inhibits angiogenesis in vitro and in vivo. Exp Cell Res 317: 1677-1688, 2011.

17. Perera Y, Farina HG, Hernandez I, et al: Systemic administration of a peptide that impairs the protein kinase (CK2) phosphorylation reduces solid tumor growth in mice. Int J Cancer 122: 57-62, 2008.

18. Perea SE, Reyes O, Baladron I, et al: CIGB-300, a novel proapoptotic peptide that impairs the CK2 phosphorylation and exhibits anticancer properties both in vitro and in vivo. Mol Cell Biochem 316: 163-167, 2008.

19. Solares AM, Santana A, Baladron I, et al: Safety and preliminary efficacy data of a novel casein kinase 2 (CK2) peptide inhibitor administered intralesionally at four dose levels in patients with cervical malignancies. BMC Cancer 9: 146, 2009.

20. Soriano-García JL, López-Díaz A, Solares-Asteasuainzarra M, et al: Pharmacological and safety evaluation of CIGB-300, a casein kinase 2 inhibitor peptide, administered intralesionally to patients with cervical cancer stage IB2/II. J Cancer Res Ther 1: 163-173, 2013

21. Perea SE, Baladron I, Garcia Y, et al: CIGB-300, a synthetic peptide-based drug that targets the CK2 phosphoaceptor domain. Translational and clinical research. Mol Cell Biochem 356 45-50, 2011.

22. Al-Lazikani B, Banerji U and Workman P: Combinatorial drug therapy for cancer in the post-genomic era. Nat Biotechnol 30 679-692, 2012

23. Chou TC: Theoretical basis, experimental design, and computerized simulation of synergism and antagonism in drug combination studies. Pharmacol Rev 58: 621-681, 2006.
24. Boyd M: The NCI In Vitro Anticancer Drug Discovery Screen. Concept, Implementation, and Operation, 1985-1995. In: Anticancer Drug Development Guide: Preclinical Screening, Clinical Trials, and Approval. Humana Press, Totowa, NJ, USA, 1997.

25. Chou TC and Hayball MP: CalcuSyn for Windows: multiple-drug dose effect analyzer and manual. Biosoft, Cambridge (UK), 1997.

26. Kitano H: Cancer as a robust system: implications for anticancer therapy. Nat Rev Cancer 4: 227-235, 2004.

27. Dancey JE and Chen HX: Strategies for optimizing combinations of molecularly targeted anticancer agents. Nat Rev Drug Discov 5: 649-659, 2006

28. Marupudi NI, Han JE, Li KW, Renard VM, Tyler BM and Brem H: Paclitaxel: a review of adverse toxicities and novel delivery strategies. Expert Opin Drug Saf 6: 609-621, 2007.

29. Gianni L, Kearns CM, Giani A, et al: Nonlinear pharmacokinetics and metabolism of paclitaxel and its pharmacokinetic/pharmacodynamic relationships in humans. J Clin Oncol 13: 180-190, 1995.

30. Andersson A, Fagerberg J, Lewensohn R and Ehrsson $\mathrm{H}$ : Pharmacokinetics of cisplatin and its monohydrated complex in humans. J Pharm Sci 85: 824-827, 1996.

31. Kuenen BC, Rosen L, Smit EF, et al: Dose-finding and pharmacokinetic study of cisplatin, gemcitabine, and SU5416 in patients with solid tumors. J Clin Oncol 20: 1657-1667, 2002.

32. Mayer LD and Janoff AS: Optimizing combination chemotherapy by controlling drug ratios. Mol Interv 7: 216-223, 2007.

33. Ohga T, Uchiumi T, Makino Y, Koike K, Wada M, Kuwano M and Kohno K: Direct involvement of the Y-box binding protein YB-1 in genotoxic stress-induced activation of the human multidrug resistance 1 gene. J Biol Chem 273: 5997-6000, 1998.

34. Hennessy M and Spiers JP: A primer on the mechanics of P-glycoprotein the multidrug transporter. Pharmacol Res 55: $1-15,2007$.

35. Gaudreault I, Guay D and Lebel M: YB-1 promotes strand separation in vitro of duplex DNA containing either mispaired bases or cisplatin modifications, exhibits endonucleolytic activities and binds several DNA repair proteins. Nucleic Acids Res 32: 316-327, 2004.

36. Skabkin MA, Evdokimova V, Thomas AA and Ovchinnikov LP The major messenger ribonucleoprotein particle protein $\mathrm{p} 50$ (YB-1) promotes nucleic acid strand annealing. J Biol Chem 276: 44841-44847, 2001.

37. Townsend DM and Tew KD: The role of glutathione-S-transferase in anti-cancer drug resistance. Oncogene 22: 7369-7375, 2003.

38. Pinna LA : Protein Kinase CK2. Wiley-Blackwell (ed). John Wiley \& Sons, Inc., 2013.

39. Drygin D, Bliesath J, Ho C, et al: CX-4945, a novel small molecule inhibitor of CK2 protein kinase, reduces hyperactivated Akt signaling and synergizes with Akt inhibitors in breast cancer cells. 21st AACR-NCI-EORTC Symposium on Molecular Targets and Cancer Therapeutics, Boston, MA. Mol Cancer Ther 8: C198, 2009.

40. Bliesath J, Huser N, Omori M, et al: Combined inhibition of EGFR and CK2 augments the attenuation of PI3K-Akt-mTOR signaling and the killing of cancer cells. Cancer Lett 322: 113-118, 2012.

41. Siddiqui-Jain A, Bliesath J, Macalino D, et al: CK2 inhibitor CX-4945 suppresses DNA repair response triggered by DNA-targeted anticancer drugs and augments efficacy: mechanistic rationale for drug combination therapy. Mol Cancer Ther 11: 994-1005, 2012.

42. Siddiqui-Jain A, Streiner N, Bliesath J, et al: CX-4945, an inhibitor of protein kinase CK2, potentiates the antitumor activity of platinum chemotherapy in models of ovarian cancer by preventing phosphorylation of XRCC1 and MDC1 and disrupting DNA damage repair. Cancer Res 71: 5494, 2011. 